\title{
Postmortem Alzheimer's Disease Hippocampi Show Oxidative Phosphorylation Gene Expression Opposite that of Isolated Pyramidal Neurons
}

\author{
Ann C. Rice ${ }^{\mathrm{a}, \mathrm{b}, *}$, Amy C. Ladd ${ }^{\mathrm{a}}$ and James P. Bennett Jra,b,c,d \\ a Parkinson's and Movement Disorders Center, Virginia Commonwealth University, Richmond, VA, USA \\ ${ }^{\mathrm{b}}$ Department of Neurology, Virginia Commonwealth University, Richmond, VA, USA \\ ${ }^{\mathrm{c}}$ Department of Psychiatry, Virginia Commonwealth University, Richmond, VA, USA \\ ${ }^{\mathrm{d}}$ Department of Physiology \& Biophysics, Virginia Commonwealth University, Richmond, VA, USA
}

Accepted 12 January 2015

\begin{abstract}
Causes of initiation and progression of sporadic Alzheimer's disease (sAD) are likely multiple and include impairment of mitochondrial bioenergetics. We analyzed RNA expression levels of multiple mitochondrial oxidative phosphorylation (OXPHOS) and biogenesis (mitobiogenesis) genes in unfixed hippocampal (WH) frozen sections (10 sAD; 9 CTL) and lasercaptured hippocampal pyramidal neurons (PyNs, $\sim 1000$ neurons from each case) from 8 sAD and 7 CTL cases. Nuclear-encoded OXPHOS genes in WH were significantly increased in SAD, whereas in isolated SAD PyNs, these same genes were significantly decreased. Mitochondrial DNA-encoded genes were increased in SAD PyNs but showed a non-significant downward trend in SAD WH. Relationships among WH and PyN gene expression levels in SAD distributed in a different population compared to CTL. Principal component analysis (PCA) revealed clustering of CTL but widespread heterogeneity of sAD samples. In SAD, mitochondrial bioenergetics at the gene expression level are depressed in vulnerable PyNs. PCA revealed that CTL samples clustered together, whereas SAD samples varied widely. From the perspective of OXPHOS bioenergetics, SAD is a heterogeneous syndrome and not likely due to a single abnormality. Increased stimulation of nuclear-encoded OXPHOS gene expression in PyNs is a rational therapeutic approach for most but not all cases of sAD.
\end{abstract}

Keywords: Laser capture microdissection, mitochondria, mitochondrial biogenesis, relative gene expression, quantitative PCR

\section{INTRODUCTION}

Alzheimer's disease (AD) is a devastating, progressive neurodegenerative disease affecting memory and cognitive function. AD incidence increases with aging and is the most common neuropathological cause of dementia in adults. Decreased glucose utilization in brain regions involved in memory and cognitive

\footnotetext{
*Correspondence to: Ann C. Rice, PhD, PO Box 980599, Department of Neurology, Virginia Commonwealth University, Richmond, VA 23298-0599, USA. Tel.: +1 804828 9664; E-mail: acrice@vcu.edu.
}

function, as detected by fluorodeoxyglucose PET scan, has been identified as an important biomarker that correlates well with level of cognitive impairment [1-4]. Along with decreased glucose utilization, numerous studies have demonstrated mitochondrial dysfunction in $\mathrm{AD}$ (reviewed in [5-13]). Abnormal mitochondrial biogenesis signaling has also been demonstrated in tissue from AD cases [14-16].

Mitochondria have multiple copies of their own DNA (mtDNA), which encode 13 oxidative phosphorylation (OXPHOS) genes, 2 rRNAs, and 22 tRNAs. The remaining $\sim 1500$ proteins found in the mitochondrial proteome are encoded in the nucleus, with only 
$\sim 80$ being part of OXPHOS complexes [17]. Mitochondrial biogenesis, a complex signaling cascade, ensures these proteins are generated in correct ratios.

Mitochondrial biogenesis is the process that increases mitochondrial mass [18-20]. Briefly, mitochondrial biogenesis is believed to begin with the transcriptional or post-translational activation of the "master regulator" $\mathrm{PGC} 1 \alpha$, which co-activates multiple genes involved in energy metabolism. These include the nuclear respiratory transcription factors NRF1 and NRF2 and estrogen-related receptor alpha $(\mathrm{ERR} \alpha) . \mathrm{NRF} 1, \mathrm{NRF} 2$, and $\mathrm{ERR} \alpha$ then initiate transcription of the nuclear-encoded respiratory genes, antioxidant genes, and mitochondrial transcription factor A (TFAM). TFAM mediates transcription of the mitochondrial DNA-encoded respiratory genes and is involved in maintenance of the mitochondrial DNA copy number.

One of the difficulties with understanding the pathogenesis pathways involved in a neurodegenerative disease such as $\mathrm{AD}$ is the limitation of currently available animal models. We chose to study AD in postmortem brain regions and then plan to identify models that mimic our findings to allow better characterization of the role of mitochondria in AD pathogenesis. The approach of studying postmortem tissue is confounded by the fact that many studies have evaluated gene expression in whole AD brain tissues, which may not accurately reflect gene expression in the specific cell types which are dysfunctional or dying, particularly in complex organs like the brain, where adjacent cells can have different functions. The cell types lost in $\mathrm{AD}$ (neurons) are not the major cell type from most brain tissues studied that are $\sim 80 \%$ glial [21]. In this report, we compared gene expression profiles of genes involved in mitochondrial function from both whole hippocampal (WH) sections and laser capture microdissected (LCM) hippocampal pyramidal neurons (PyNs) from $\mathrm{AD}$ and control (CTL) postmortem tissue. Because of our historical interest in mitochondrial pathophysiology of neurodegenerative diseases, we focused on regulation of mitochondrial gene expression and mitobiogenesis signaling.

\section{MATERIALS AND METHODS}

\section{Tissue}

Postmortem flash frozen hippocampal brain tissue was obtained from the Brain Resource Facility at the University of Virginia. Tissue was embedded in cryostat mounting media and sectioned at $10 \mu \mathrm{m}$.

\section{Whole hippocampal RNA isolation/cDNA}

Approximately 10 cryostat sections from each hippocampus were processed for RNA extraction using the miRNeasy kit following the manufacturer's instructions (Qiagen). RNA integrity (RNA quality index, RQI) was assessed using BioRad Experion capillary electrophoresis technology and software (RQI $8.49 \pm 0.78$ (mean $\pm \mathrm{SD})$ ). RNA was converted to cDNA using the iScript cDNA synthesis kit for RT-qPCR following the manufacturer's instructions (BioRad). Cases consisted of 10 AD (mean age $79.9 \pm 9 \mathrm{y}$; mean postmortem interval $6 \pm 4.5 \mathrm{~h} ; 4 \mathrm{M}$; $6 \mathrm{~F}$ ) and $9 \mathrm{CTL}$ (mean age $61.8 \pm 15 \mathrm{y}$; mean postmortem interval $8 \pm 4.5 \mathrm{~h} ; 3 \mathrm{M} ; 6 \mathrm{~F})$. While these age differences are significantly different, when we compared age with gene expression levels for the 76 mitochondrial related genes presented, only two were significantly correlated with age (Supplementary Table 1).

\section{Laser capture microdissection/RNA isolation/cDNA}

Slices were fixed in $70 \%$ EtOH for $1 \mathrm{~min}$, hydrated, stained with Histogene (Arcturus), washed, dehydrated (10 dips each), and cleared in xylene (4 min). Hippocampal PyNs were identified morphologically and captured using the Arcturus XT laser capture system. Multiple caps per case were pooled to obtain RNA from approximately 1000 cells. RNA was extracted using the Qiagen RNeasy Plus Micro kit following the manufacturer's instructions. RNA integrity was assessed as above (RQI $6.8 \pm 1.8$ (mean $\pm \mathrm{SD})$ ). RNA was preamplified and reverse transcribed using the kit from Nugen (Ovation PicoSL WTA system v2) following manufacturer's instructions as we have done previously [22]. Samples were diluted to $0.2-0.25 \mathrm{ng} / \mu \mathrm{l}$ for quantitative PCR (qPCR) analysis, because there was inhibition of the PCR at higher concentrations. Cases consisted of $8 \mathrm{AD}$ (mean age $80 \pm 5.5 \mathrm{y}$; mean postmortem interval $4.5 \pm 3.7 \mathrm{~h} ; 3 \mathrm{M} ; 5 \mathrm{~F}$ ) and $7 \mathrm{CTL}$ (mean age $57.7 \pm 13 \mathrm{y}$; mean post-mortem interval $8.7 \pm 4.6 \mathrm{~h} ; 2 \mathrm{M} ; 5 \mathrm{~F})$. While these age differences are significantly different, when we compared age with gene expression levels for the 75 mitochondrial related genes presented, only seven were significantly correlated with age (Supplementary Table 1). Four samples with the RNA integrity numbers below 8 from whole tissue, were not processed for LCM (two AD and two CTL), since we believe the drop in RNA integrity that occurs with the additional processing of LCM samples 
would result in very low RNA integrity values and produce unreliable results [23].

\section{Quantitative PCR (qPCR)}

qPCR was used to determine the relative gene expression of multiple genes. Multiplex qPCR was used for mitochondrial-encoded genes (ND4, ND2, COX III, and $12 \mathrm{~S}$ rRNA); EvaGreen (BioRad) qPCR was used for mitobiogenesis genes (PGC1 $\alpha$, NRF1, NRF2, ERR $\alpha$, Pol $\gamma$, and TFAM), neuron and glia specific genes ( $\beta$-tubulin, MAP2, neurofilament-L, GFAP, and glutamate transporter-1) and reference gene expression (GAPDH, CYC1, 14-3-3z, B2M, UBC, and TOPO1). Primers were designed using latest updates of Beacon Designer software (Premier Biosoft, current version 8.1.2). Nuclear-encoded OXPHOS relative gene expression was determined using SABiosciences' mitochondrial energy metabolism plus array plates (PAHS-008Y) following the manufacturer's instructions. GeNorm was used to select three reference genes with the least variability across all samples (14-3-3z, GAPDH, and CYC1) to normalize $\mathrm{qPCR}$ data. Statistical analyses were performed using QBase+ (Biogazelle), Prism (GraphPad), and Excel (Microsoft). Principal component analysis used the XLSTAT plugin for Excel.

\section{RESULTS}

To demonstrate neuronal enrichment in LCM tissue, three neuronal specific genes and two glial specific genes were analyzed by qPCR. In WH samples, the relative mean expression of glial markers/neuronal markers was $312 \%$ compared to $7 \%$ in LCM isolated PyNs. The relative mean expression of neuronal markers/glial markers was 1,390\% in LCM isolated PyNs compared to $32 \%$ in whole hippocampi. These data (not shown) indicate our LCM tissue is enriched in neuronal phenotype-expressing cell types.

Relative gene expression profiles of nuclearencoded OXPHOS genes from WH tissue and LCM isolated PyNs are shown in Fig. 1 grouped by OXPHOS complex. In our cohort of tissue samples, the WH profiles showed significantly elevated differences in gene expression from AD compared to CTL cases (left panel; 2-way ANOVA $p \leq 0.0001$, graphs A, C, and G, $p=0.0002$ graph E). Whereas, in LCM isolated PyNs, the OXPHOS gene expression profile was significantly reduced in $\mathrm{AD}$ compared to $\mathrm{CTL}$ for all complexes (right panel; 2-way ANOVA $p<0.0001$, graphs B, D, $\mathrm{F}$, and $\mathrm{H})$.
The relative expression of the mitochondrialencoded genes from $\mathrm{WH}$ and LCM isolated PyNs is depicted in Fig. 2. In LCM isolated PyNs, there is a significant increase in expression of mitochondrial-encoded genes in AD cases (2-way ANOVA $p=0.0001$, graph $\mathrm{B}$ ), while in WH there is not a significant difference between $\mathrm{AD}$ compared to CTL cases (graph A), although the trend was toward decreased expression (2-way ANOVA $p=0.1963$ ).

The relative expression of genes involved in mitochondrial biogenesis is shown in Fig. 3. There were no differences in gene expression between $\mathrm{AD}$ and $\mathrm{CTL}$ in either WH or in LCM isolated PyNs (2-way ANOVA $p=0.2614$ and $p=0.1365$, respectively). There was an interesting trend observed with PGC $1 \alpha$, which showed a decreased trend in $\mathrm{WH}$ and increased trend in isolated PyNs.

Figure 4 shows that the relationships among OXPHOS gene expression levels in WH compared to PyNs distributed into two apparently different populations in sporadic AD (sAD) compared to CTL samples. To explore this apparent difference further, we carried out principal component analysis (PCA) of the WH/pyramidal neuron ratios for OXPHOS genes in SAD $(n=8)$ and CTL $(n=7)$ cases. Figure 5 presents the PCA results and shows that the CTL samples clustered together, whereas the SAD samples were heterogeneously distributed and did not cluster. Two of the sAD cases showed PCA localizations within the CTL cluster, but the other six SAD cases varied widely in their locations both in relationship to CTL and each other.

\section{DISCUSSION}

There are three major findings of our study. First, we observed that hippocampal PyNs isolated from AD hippocampi using LCM have reduced expression of nuclear DNA-encoded OXPHOS genes compared to CTL, while the corresponding WH showed significantly increased expression. We pooled extracts from $\sim 1000$ neurons from each hippocampus and did not classify them according to either "CA" (cornu Ammonis) region or presence/absence of neurofibrillary tangles (that would generally be minimal in CTL). Thus our data represent population statistical means for each hippocampal specimen. Because neurons account for $\sim 70 \%$ or more of 2DG uptake [24], and since PyNs are a major neuronal component of hippocampi, our findings may account for part of the decline in hippocampal ${ }^{18} \mathrm{~F}-2 \mathrm{DG}-\mathrm{PET}$ signal that predicts conversion to $\mathrm{AD}[25]$. 

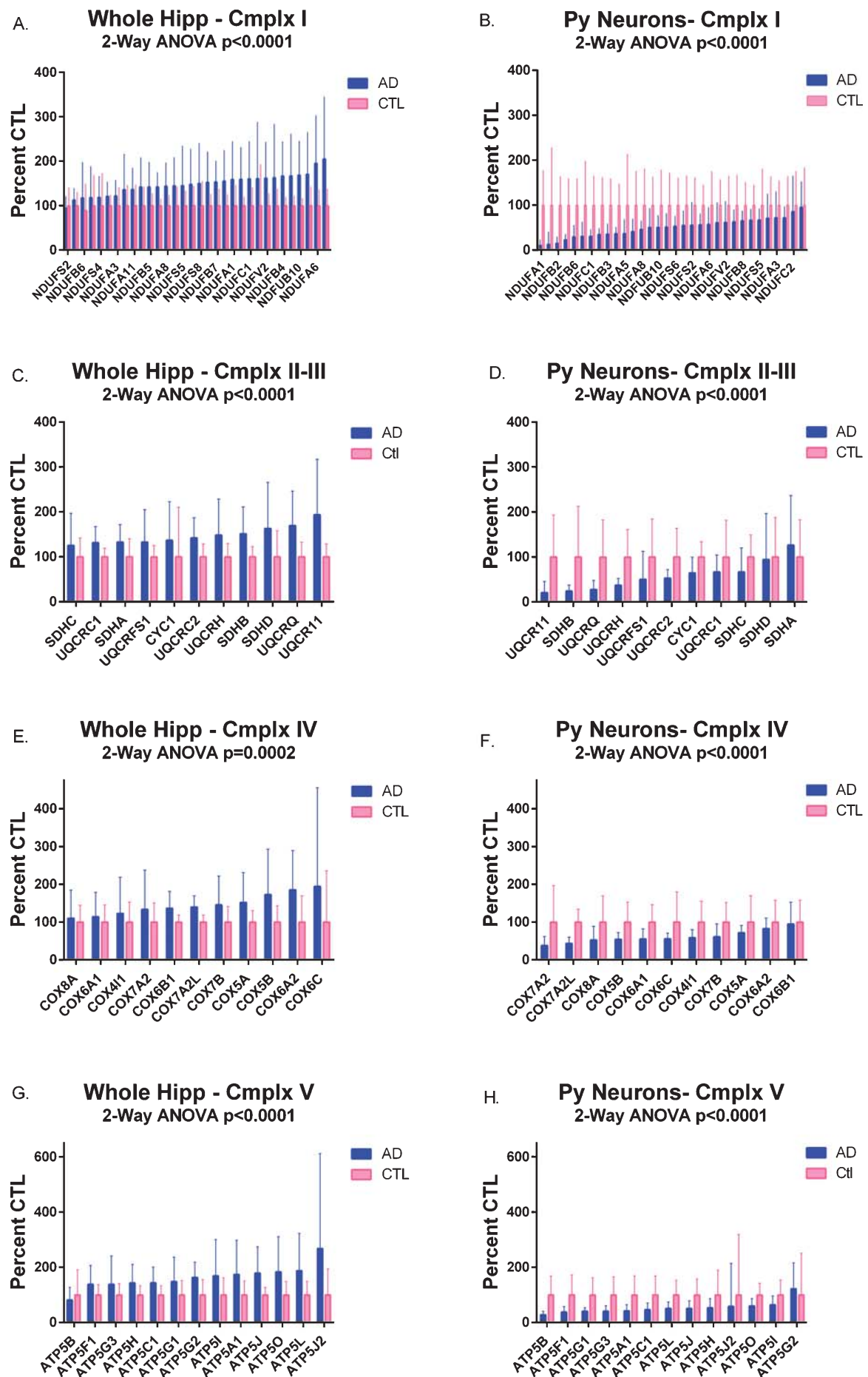

Fig. 1. Relative gene expression of nuclear-encoded OXPHOS genes from whole hippocampi (A, C, E, and G) and laser captured pyramidal (Py) neurons (B, D, F, and H). Samples were analyzed by 2-way ANOVA for each complex for type of sample. For each complex, there is a significant increase in expression in AD in whole tissue and a significant decrease in expression in AD in isolated hippocampal Py neurons $(p<0.0001$ for graphs A-D and F-H; $p=0.0002$ graph E). 

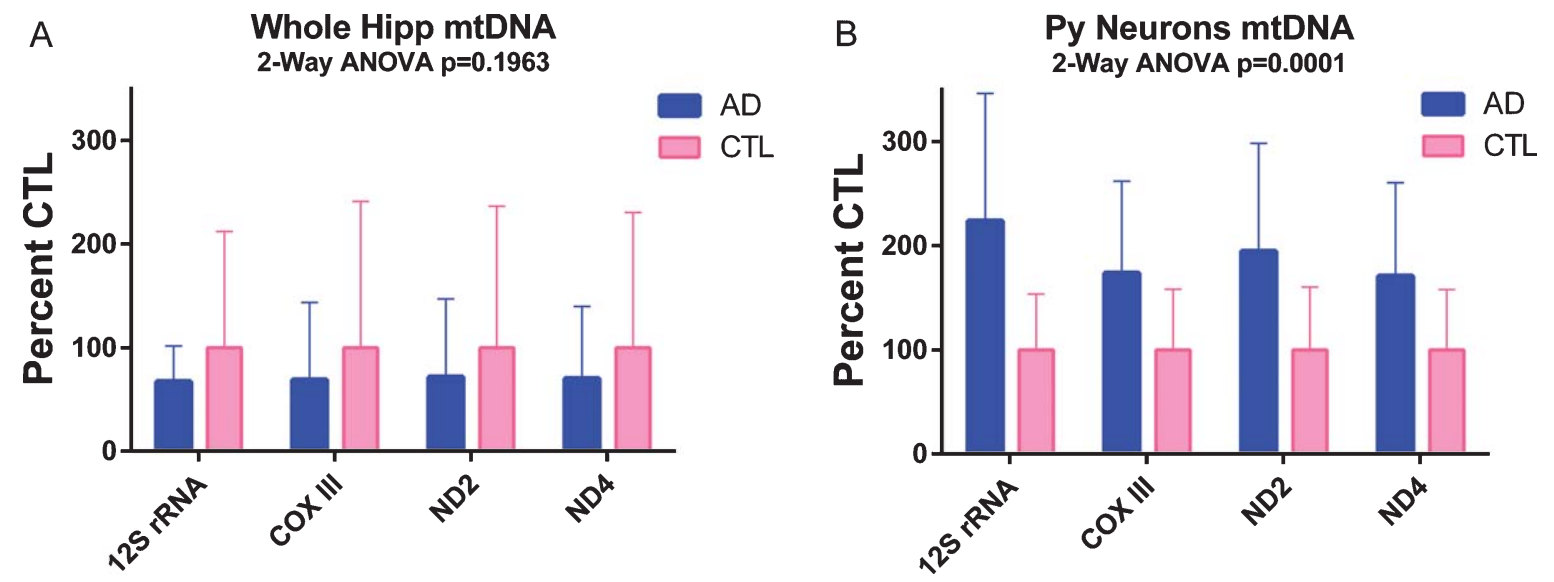

Fig. 2. Relative gene expression of mitochondrial-encoded genes from whole hippocampi (A) and laser captured pyramidal (Py) neurons (B). Samples were analyzed by 2-way ANOVA for each type of sample. Whole tissue samples were not significantly different $(p=0.1963)$, whereas isolated Py neurons showed a significant increase in expression $(p=0.0001)$.
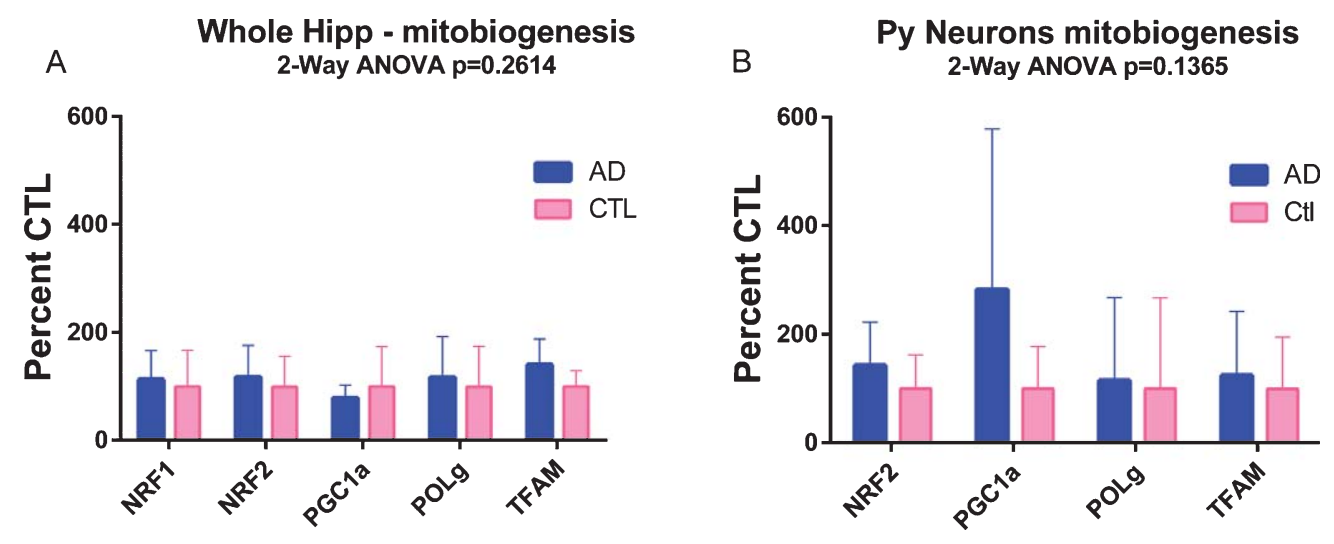

Fig. 3. Relative gene expression of mitochondrial biogenesis genes from whole hippocampi (A) and laser captured pyramidal (Py) neurons (B). Samples were analyzed by 2-way ANOVA for each type of sample. There were no significant difference either in whole tissue $(p=0.2614)$ or in isolated Py neurons $(p=0.1365)$.

The second major finding is that LCM isolated PyNs from AD brains, compared to CTL, showed increased mitochondrial gene expression. It could be that our LCM isolated PyNs are trying to upregulate ATP production by increasing mitochondrial gene expression, while some feedback mechanism telling the nucleus to upregulate expression is not working correctly. Since our LCM samples could not be processed with greater amounts of starting material, better evaluation of the expression of mitochondrial biogenesis genes, which are not highly expressed in these samples, will be the focus of future studies, so we can begin to understand the metabolic changes observed in AD. Additionally, LCM material is localized to the peri-nuclear area and probably accurately reflects nuclear DNA expression, but with mitochondria spread throughout neuronal processes there may be differences we have not detected.
Liang et al. [26] also showed decreased expression of nuclear-encoded respiratory genes in LCM isolated hippocampal PyNs from AD compared to CTL. Using LCM isolated neurons from multiple brain regions and microarray analyses, they reported decreases in respiratory gene expression by $70 \%$ in posterior cingulate cortex, $65 \%$ in middle temporal gyrus, and $61 \%$ in hippocampus. Additional studies by this group characterized gene expression profiles from specific types of neurons in multiple brain regions using LCM in control tissue [27] and in $\mathrm{AD}[28,29]$. Another group that used LCM to evaluate gene expression changes in hippocampal PyNs found genes involved with endosomal trafficking were elevated, while neurotrophin receptors were decreased [30].

Several studies have compared gene or protein expression profiles of specific cell types isolated by LCM to the whole tissue surrounding the cells of interest 


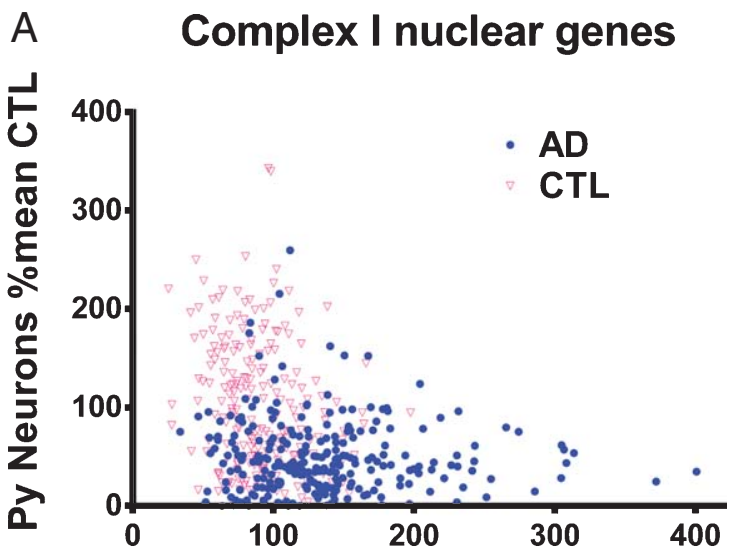

Whole Hipp \%mean CTL

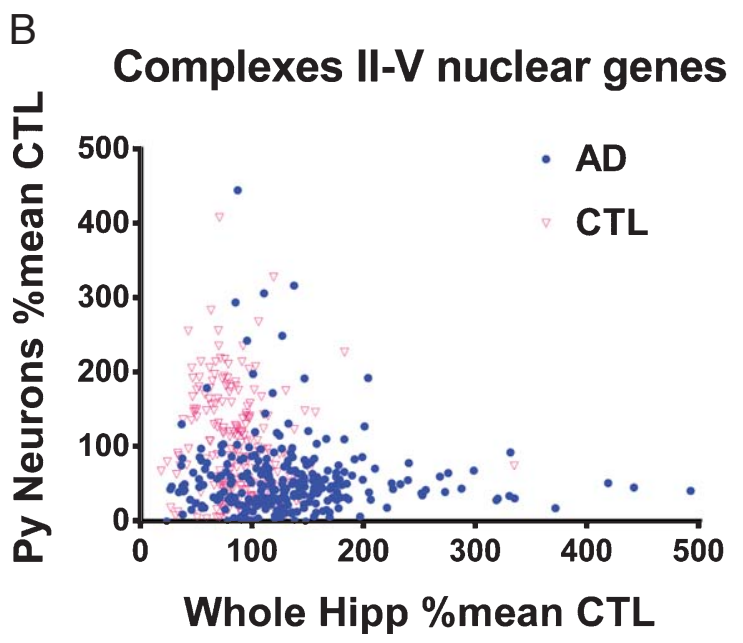

Fig. 4. Graphs of the ratios of gene expression for nuclear Complex I genes (top) and nuclear Complexes II-V genes (bottom), expressed as the ratios of expression in hippocampal sections/expression in isolated hippocampal pyramidal (Py) neurons. All expression levels are presented as \% mean CTL values. CTL samples are shown in red and SAD samples are shown in blue.

and found different expression profiles. This probably occurs because the cells of interest are diluted out in the whole tissue samples. In the cancer field, the expression profile of specific tumors is critical to the treatment paradigm. Silvestri et al. [31] isolated cancer epithelium separate from stroma and compared to whole tissue from colorectal cancer samples and found very different profiles. LCM isolated spinal cord motor neurons from amyotrophic lateral sclersosi cases have a different gene expression profile than the whole spinal ventral horn [32]. These studies and ours support the need to evaluate specific cell types when trying to understand a
Principal Component Analysis

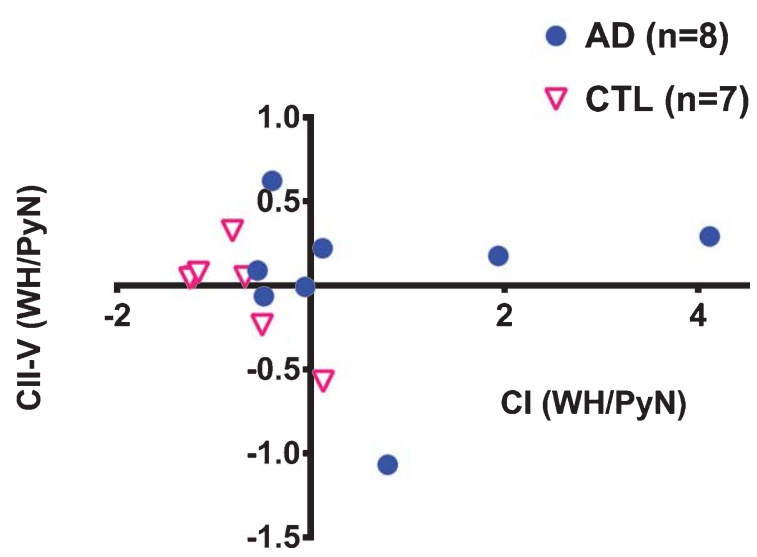

Fig. 5. Principal component analysis (PCA) of control (CTL) and sporadic Alzheimer's disease (sAD) OXPHOS gene expression levels. For each CTL $(n=7)$ and $\operatorname{sAD}(n=8)$ case, the ratios of whole hippocampal (WH)/pyramidal neurons (PyNs) for all nuclear Complex I genes or all nuclear Complex II-V genes were averaged. PCA was carried out for these averaged ratios in each CTL or SAD case using the XLSTAT plugin for Excel. Shown are the results for clustering of CTL cases and lack of clustering for SAD cases.

disease phenotype, even though these studies are more tedious and difficult to perform.

LCM enriches a specific cell type, and some quality control measures are a good practice. We chose to demonstrate enrichment of neurons with qPCR and relative expression levels of neuronal and glial specific markers. In the series of publications by Liang et al. [27-29] who performed microarray analysis of LCM neurons, they excluded samples with high GFAP levels. Another study that isolated astrocytes, oligodendrocytes, and microglia performed PCR for GFAP, olig 2, and CD68 to demonstrate enrichment of each cell type, respectively [23].

Multiple studies using microarray analysis have described whole tissue expression of metabolic genes in AD compared to CTL [33-37]. Hippocampal expression of metabolism-associated genes was reported to be significantly decreased $[33,34]$ in contrast to our findings of nuclear-encoded OXPHOS gene expression being elevated in whole tissue. Both studies were microarray studies and had relatively small sample sizes (like ours), which could contribute to the differences observed between our data and theirs. One group had six AD with varying levels of disease severity [34] and the other group used $22 \mathrm{AD}$ and nine CTL with the $\mathrm{AD}$ cases split between mild, moderate, and severe [33]. Berchtold et al. [35] also used microarray data and evaluated multiple brain regions including the hippocampus between $\mathrm{AD}$, mild cognitive 
impairment, and control (determined by Mini-Mental State Examination score and Braak score). They found an increase in respiratory gene expression in the mild cognitive impairment group compared to either AD or control and a decrease in respiratory gene expression in $\mathrm{AD}$ compared to control. We do not have data on the severity of the disease condition for our cases, but all were diagnosed by histopathology as AD. Additionally, the Berchtold group had a larger sample size (16-27/group) than the other studies ([33, 34] and present study).

Other groups that used whole tissue evaluated expression by qPCR, western blot, immunohistochemistry, or in situ hybridization and compared AD to control [14-16, 38, 39]. Hirai et al. [38] show decreased mitochondria, with increased mitochondrial DNA that localized to lipofuscin vesicles and increased cytochrome oxidase in hippocampal PyNs from AD cases. Sheng et al. [14] demonstrated decreased protein levels of key proteins involved in mitochondrial biogenesis (PGC1 $\alpha$, NRF 1, NRF2, and TFAM) in hippocampi from AD cases. Young-Collier et al. [15] also showed decreased expression of mitochondrial biogenesis genes (qPCR) in frontal cortex of AD cases compared to control, as well as decreased mitochondrial mass (porin), even though relative levels of most respiratory proteins assessed by western blot were not different from control.

In a previous study we demonstrated loss of the mitochondrial biogenesis expression hierarchy in $\mathrm{AD}$ compared to control [16], although the overall mitobiogenesis gene expression levels were not significantly different in WH between $\mathrm{AD}$ and control (Fig. 3). Manczak et al. [39] used punch biopsies from frontal cortex and qPCR to demonstrate differential expression of mitochondrial-encoded respiratory genes with complex I decreased and complexes IV and V increased. Our analysis of mitochondrial gene expression involved evaluating four different genes around the mitochondrial genome, since a polycistronic message is transcribed, instead of representative genes from each complex. Three of the genes (ND2, ND4, and Cox III) were expressed relatively equally and the fourth gene (12 S rRNA) was expressed at a greater level as expected for rRNAs. However, we did not observe differences in expression level between $\mathrm{AD}$ and control from our WH samples.

The third major finding of our study, from the perspective of hippocampal OXPHOS bioenergetics, is that the there is more variability in the WH/PyN expression level ratios in the SAD populations compared to the CTL populations. The PCA findings indicate our samples distribute into two distinct populations with the CTL cases having a tighter grouping and indicating our sAD cases are a more heterogeneous population. This could mean SAD is not a single process, with the prediction that therapies enhancing OXPHOS gene expression in vulnerable PyNs are predicted to have variable efficacy. Ideally, a reliable biomarker of OXPHOS status in early AD would predict therapeutic response to increasing hippocampal PyN OXPHOS bioenergetics.

We realize other variables are at play that could increase the variability such as postmortem interval and age, however these values are actually tighter (smaller SDs) in the AD cases compared to the CTL cases and would tend to increase the variability of the CTL group.

There are several limitations of our study. First, we had a limited number of samples. Since AD afflicts $\sim 5$ million Americans, it is impossible to project our findings from a few AD and CTL cases to the AD population at large. Second, we evaluated only OXPHOS gene expression and did not assay mitochondrial physiology such as respiration or biochemistry such as electron transport chain protein levels. While such assays are possible in hippocampal sections, they are not yet feasible in the LCM isolated neuronal samples we compared to intact hippocampi. Thus, our results need to be considered in the context of gene expression only measurements. Third, particularly for LCM PyNs, we are assaying "survivors", PyNs present in hippocampi of persons who died with AD. It is not known whether these surviving neurons reflect at all changes in cells that already died, or whether these surviving neurons possess special properties accounting for their survival. This is a common problem with all postmortem studies of neurodegenerative diseases. In the case of $\mathrm{AD}$, it is theoretically possible to carry out such studies in brains from persons who died with mild cognitive impairment, a frequent prodromal condition prior to overt dementia and AD pathology. Fourth, we did not try to remove PyNs from specific CA hippocampal areas, or select those burdened with neurofibrillary tangles in the AD cases. For the latter, it would be necessary to find an approach that would preserve RNA quality while allowing detection of neurofibrillary tangles. Potentially, fluorescent staining with Thioflavin-S could allow such an analysis, as was done by Liang et al. [26, 28, 29].

In conclusion, we find that AD hippocampal PyNs are metabolically compromised in terms of their potential for mitochondrial bioenergetic OXPHOS function. We also show that LCM is capable of capturing 
neuronally enriched cell populations. We speculate that this bioenergetic impairment in vulnerable AD PyNs increases their risk of dysfunction and dying, but we do not yet know if this metabolic impairment is more primary in the pathogenesis of $\mathrm{AD}$ neurodegeneration or is a secondary epiphenomenon. Finally, from the perspective of PCA analysis of OXPHOS bioenergetics, we observe that SAD is a markedly heterogeneous condition relative to CTL samples that are much more homogeneous. Therapies to increase OXPHOS bioenergetics in vulnerable PyNs may not benefit all sAD patients.

\section{ACKNOWLEDGMENTS}

This research was supported by VCU's Parkinson's and Movement Disorders Center.

Authors' disclosures available online (http://j-alz. com/manuscript-disclosures/14-2937r1).

\section{SUPPLEMENTARY MATERIAL}

The supplementary material is available in the electronic version of this article: http://dx.doi.org/ 10.3233/JAD-142937.

\section{REFERENCES}

[1] Mosconi L, Mistur R, Switalski R, Tsui WH, Glodzik L, Li Y, Pirraglia E, De Santi S, Reisberg B, Wisniewski T, de Leon MJ (2009) FDG-PET changes in brain glucose metabolism from normal cognition to pathologically verified Alzheimer's disease. Eur J Nucl Med Mol Imaging 36, 811-822.

[2] Jagust WJ, Landau SM, Shaw LM, Trojanowski JQ, Koeppe RA, Reiman EM, Foster NL, Petersen RC, Weiner MW, Price JC, Mathis CA, Alzheimer's Disease Neuroimaging I (2009) Relationships between biomarkers in aging and dementia. Neurology 73, 1193-1199.

[3] Herholz K, Westwood S, Haense C, Dunn G (2011) Evaluation of a calibrated (18)F-FDG PET score as a biomarker for progression in Alzheimer disease and mild cognitive impairment. J Nucl Med 52, 1218-1226.

[4] Landau SM, Harvey D, Madison CM, Koeppe RA, Reiman EM, Foster NL, Weiner MW, Jagust WJ, Alzheimer's Disease Neuroimaging I (2011) Associations between cognitive, functional, and FDG-PET measures of decline in AD and MCI. Neurobiol Aging 32, 1207-1218.

[5] Gibson GE, Shi Q (2010) A mitocentric view of Alzheimer's disease suggests multi-faceted treatments. J Alzheimers Dis 20(Suppl 2), S591-S607.

[6] Coskun PE, Wyrembak J, Derbereva O, Melkonian G, Doran E, Lott IT, Head E, Cotman CW, Wallace DC (2010) Systemic mitochondrial dysfunction and the etiology of Alzheimer's disease and down syndrome dementia. J Alzheimers Dis 20(Suppl 2), S293-S310.

[7] Selfridge JE, E L, Lu J, Swerdlow RH (2013) Role of mitochondrial homeostasis and dynamics in Alzheimer's disease. Neurobiol Dis 51, 3-12.
[8] Swerdlow RH, Parks JK, Cassarino DS, Maguire DJ, Maguire RS, Bennett JP Jr, Davis RE, Parker WD Jr (1997) Cybrids in Alzheimer's disease: A cellular model of the disease? Neurology 49, 918-925.

[9] Muller WE, Eckert A, Kurz C, Eckert GP, Leuner K (2010) Mitochondrial dysfunction: Common final pathway in brain aging and Alzheimer's disease-therapeutic aspects. $\mathrm{Mol} \mathrm{Neu-}$ robiol 41, 159-171.

[10] Horan MP, Pichaud N, Ballard JW (2012) Review: Quantifying mitochondrial dysfunction in complex diseases of aging. J Gerontol A Biol Sci Med Sci 67, 1022-1035.

[11] Johri A, Beal MF (2012) Mitochondrial dysfunction in neurodegenerative diseases. J Pharmacol Exp Ther 342, 619-630.

[12] Karbowski M, Neutzner A (2012) Neurodegeneration as a consequence of failed mitochondrial maintenance. Acta Neuropathol 123, 157-171.

[13] Caldeira GL, Ferreira IL, Rego AC (2013) Impaired transcription in Alzheimer's disease: Key role in mitochondrial dysfunction and oxidative stress. J Alzheimers Dis 34, 115131.

[14] Sheng B, Wang X, Su B, Lee HG, Casadesus G, Perry G, Zhu X (2012) Impaired mitochondrial biogenesis contributes to mitochondrial dysfunction in Alzheimer's disease. J Neurochem 120, 419-429.

[15] Young-Collier KJ, McArdle M, Bennett JP (2012) The dying of the light: Mitochondrial failure in Alzheimer's disease. $J$ Alzheimers Dis 28, 771-781.

[16] Rice AC, Keeney PM, Algarzae NK, Ladd AC, Thomas RR, Bennett JP Jr (2014) Mitochondrial DNA copy numbers in pyramidal neurons are decreased and mitochondrial biogenesis transcriptome signaling is disrupted in Alzheimer's disease hippocampi. J Alzheimers Dis 40, 319-330.

[17] Koopman WJ, Distelmaier F, Smeitink JA, Willems PH (2013) OXPHOS mutations and neurodegeneration. EMBO J 32, 929.

[18] Scarpulla RC (2008) Transcriptional paradigms in mammalian mitochondrial biogenesis and function. Physiol Rev 88, 611-638.

[19] Scarpulla RC, Vega RB, Kelly DP (2012) Transcriptional integration of mitochondrial biogenesis. Trends Endocrinol Metab 23, 459-466.

[20] Hock MB, Kralli A (2009) Transcriptional control of mitochondrial biogenesis and function. Annu Rev Physiol 71, 177-203.

[21] Walloe S, Pakkenberg B, Fabricius K (2014) Stereological estimation of total cell numbers in the human cerebral and cerebellar cortex. Front Hum Neurosci 8, 508.

[22] Ladd AC, Govind KP, Bennett MM, JP Jr (2014) Mitochondrial oxidative phosphorylation transcriptome alterations in human amyotrophic lateral sclerosis spinal cord and blood. Neuromolecular Med 16, 714-726.

[23] Waller R, Woodroofe MN, Francese S, Heath PR, Wharton SB, Ince PG, Sharrack B, Simpson JE (2012) Isolation of enriched glial populations from post-mortem human CNS material by immuno-laser capture microdissection. J Neurosci Methods 208, 108-113.

[24] Mosconi L (2013) Glucose metabolism in normal aging and Alzheimer's disease: Methodological and physiological considerations for PET studies. Clin Transl Imaging 1(4), $1-25$.

[25] Mosconi L, De Santi S, Li J, Tsui WH, Li Y, Boppana M, Laska E, Rusinek H, de Leon MJ (2008) Hippocampal hypometabolism predicts cognitive decline from normal aging. Neurobiol Aging 29, 676-692. 
[26] Liang WS, Reiman EM, Valla J, Dunckley T, Beach TG, Grover A, Niedzielko TL, Schneider LE, Mastroeni D, Caselli R, Kukull W, Morris JC, Hulette CM, Schmechel D, Rogers J, Stephan DA (2008) Alzheimer's disease is associated with reduced expression of energy metabolism genes in posterior cingulate neurons. Proc Natl Acad Sci U S A 105, 4441-4446.

[27] Liang WS, Dunckley T, Beach TG, Grover A, Mastroeni D, Walker DG, Caselli RJ, Kukull WA, McKeel D, Morris JC, Hulette C, Schmechel D, Alexander GE, Reiman EM, Rogers J, Stephan DA (2007) Gene expression profiles in anatomically and functionally distinct regions of the normal aged human brain. Physiol Genomics 28, 311-322.

[28] Liang WS, Dunckley T, Beach TG, Grover A, Mastroeni D, Ramsey K, Caselli RJ, Kukull WA, McKeel D, Morris JC, Hulette CM, Schmechel D, Reiman EM, Rogers J, Stephan DA (2008) Altered neuronal gene expression in brain regions differentially affected by Alzheimer's disease: A reference data set. Physiol Genomics 33, 240-256.

[29] Liang WS, Dunckley T, Beach TG, Grover A, Mastroeni D, Ramsey K, Caselli RJ, Kukull WA, McKeel D, Morris JC, Hulette CM, Schmechel D, Reiman EM, Rogers J, Stephan DA (2010) Neuronal gene expression in nondemented individuals with intermediate Alzheimer's disease neuropathology. Neurobiol Aging 31, 549-566.

[30] Ginsberg SD, Alldred MJ, Counts SE, Cataldo AM, Neve RL, Jiang Y, Wuu J, Chao MV, Mufson EJ, Nixon RA, Che S (2010) Microarray analysis of hippocampal CA1 neurons implicates early endosomal dysfunction during Alzheimer's disease progression. Biol Psychiatry 68, 885-893.

[31] Silvestri A, Colombatti A, Calvert VS, Deng J, Mammano E, Belluco C, De Marchi F, Nitti D, Liotta LA, Petricoin EF, Pierobon M (2010) Protein pathway biomarker analysis of human cancer reveals requirement for upfront cellular-enrichment processing. Lab Invest $\mathbf{9 0}, 787-796$.
[32] Jiang YM, Yamamoto M, Kobayashi Y, Yoshihara T, Liang Y, Terao S, Takeuchi H, Ishigaki S, Katsuno M, Adachi H, Niwa J, Tanaka F, Doyu M, Yoshida M, Hashizume Y, Sobue G (2005) Gene expression profile of spinal motor neurons in sporadic amyotrophic lateral sclerosis. Ann Neurol 57, 236251.

[33] Blalock EM, Geddes JW, Chen KC, Porter NM, Markesbery WR, Landfield PW (2004) Incipient Alzheimer's disease: Microarray correlation analyses reveal major transcriptional and tumor suppressor responses. Proc Natl Acad Sci U S A 101, 2173-2178.

[34] Brooks WM, Lynch PJ, Ingle CC, Hatton A, Emson PC, Faull RL, Starkey MP (2007) Gene expression profiles of metabolic enzyme transcripts in Alzheimer's disease. Brain Res 1127, 127-135.

[35] Berchtold NC, Sabbagh MN, Beach TG, Kim RC, Cribbs DH, Cotman CW (2014) Brain gene expression patterns differentiate mild cognitive impairment from normal aged and Alzheimer's disease. Neurobiol Aging 35, 1961-1972.

[36] Ding B, Xi Y, Gao M, Li Z, Xu C, Fan S, He W (2014) Gene expression profiles of entorhinal cortex in Alzheimer's disease. Am J Alzheimers Dis Other Demen 29, 526-532.

[37] Miller JA, Oldham MC, Geschwind DH (2008) A systems level analysis of transcriptional changes in Alzheimer's disease and normal aging. J Neurosci 28, 1410-1420.

[38] Hirai K, Aliev G, Nunomura A, Fujioka H, Russell RL, Atwood CS, Johnson AB, Kress Y, Vinters HV, Tabaton M, Shimohama S, Cash AD, Siedlak SL, Harris PL, Jones PK, Petersen RB, Perry G, Smith MA (2001) Mitochondrial abnormalities in Alzheimer's disease. J Neurosci 21, 3017-3023.

[39] Manczak M, Park BS, Jung Y, Reddy PH (2004) Differential expression of oxidative phosphorylation genes in patients with Alzheimer's disease: Implications for early mitochondrial dysfunction and oxidative damage. Neuromolecular Med 5, 147-162. 